\title{
Development and Evaluation of Decision Aid for Family Surrogate Decision Makers of Patients with Acute Kidney Injury Requiring Renal Replacement Therapy (RRT) in ICU: A Study Protocol
}

\section{Miao Zheng}

Guizhou University of Traditional Chinese Medicine https://orcid.org/0000-0003-3249-0975

\section{Changlin Yin}

First Affiliated Hospital of Army Medical University

\section{Ying Cao}

First Affiliated Hospital of Army Medical University

\section{Yonghui Zhang}

First Affiliated Hospital of Army Medical University

\section{Kuoliang Zhang}

Guizhou University of Traditional Chinese Medicine

\section{Xiaoqin Zhang}

Guizhou University of Traditional Chinese Medicine

\section{Wei Bian}

First Affiliated Hospital of Army Medical University

Lihua Wang ( $\nabla 1025982758 @ q q . c o m$ )

https://orcid.org/0000-0002-3053-4893

\section{Study protocol}

Keywords: Decision Aids, Decision Support Techniques, Family, Intensive Care Units, Renal Replacement Therapy, Shared Decision Making, Surrogate Decision Making

Posted Date: July 28th, 2020

DOl: https://doi.org/10.21203/rs.3.rs-47982/v1

License: (c) (1) This work is licensed under a Creative Commons Attribution 4.0 International License. Read Full License 


\section{Abstract}

Background Shared decision-making is endorsed by guidelines in both acute kidney injury and critical care medicine. However, there is still a huge need for effective interventions, especially focusing on decisions in renal replacement therapy for ICU patients with acute kidney injury. Decision aid is an evidence-based support to achieve shared decision-making for better decision quality, which not only enhances knowledge of treatment options but also matches patients' preferences and values.

Objectives To develop and evaluate a decision aid for family surrogate decision makers of ICU patients with acute kidney injury who are requiring renal replacement therapy, following a systematic and rigorous process.

Methods We will follow the systematic development process combined with user-centered design, to develop and evaluate the decision aid in three phases: (1) development of a decision aid based on extensive literature reviews, key stakeholders' interviews, evidence synthesis and drafting prototype; (2) alpha-testing ("near-live" usability) during simulated clinical encounters to test the comprehensibility, acceptability and usability; (3) beta-testing ("Iive" usability) to examine the feasibility in the clinical context. User-testing will be conducted for the iterative revision of the decision aid. The IPDASi (v4.0) will be used for the qualitative assessment.

Discussion This study may help family surrogate decision makers make renal replacement treatment decisions that match with their loved one's preferences and values in ICU context, while helping usercentered design integrate into this complex medical decision making.

Trial registration number Chinese Clinical Trial registry (ChiCTR2000031613).

\section{Contributions To The Literature}

- Our paper will determine the opportunities to facilitate family members of critically ill patients to engage in shared decision making with ICU clinicians.

- We will provide insights to identify family surrogate decision makers eligible to receive a decision aid and how and when to provide this decision support to them to minimize knowledge gap on ICU workflow.

- Ultimately, we will delineate an effective development process that will enable user-centered design to integrate into clinical practice to achieve the feasible and practicable decision aid.

\section{Introduction}

Acute kidney injury (AKI) is one of the most common critical syndromes in the intensive care unit (ICU), which refers to high morbidity and mortality [1]. According to recent Chinese research, $51 \%$ of critically ill patients occur AKI, which causes seven-fold mortality and increases the length of stay and healthcare costs [2]. Nowadays, renal replacement therapy (RRT) has been one of the most crucial life supports in 
ICU so that the incidence of AKI treated with RRT is increasing steeply [3, 4]. RRT is regarded as lifesustaining for AKI, with significant value in critically ill [5-7]. There is no doubt that the decision whether or not to choose RRT should be described as preference-sensitive. Actually, it is more driven by clinicianand system-level factors rather than patients' preferences and values [8]. In a recent research, $60 \%$ of patients accept RRT based on clinicians' willingness to offer treatment, while only $9 \%$ of patients treated with RRT when clinicians give unwillingness to offer treatment [9].

Many critically ill patients are sedated or in a coma, families are often involved as the extender (surrogate decision makers) to engage in treatment decision making in ICU. However, the temporary suddenness and clinical uncertainty of life-sustaining treatment often make surrogates into decisional conflict, and burden them with severe psychological syndromes such as post-traumatic stress disorder, anxiety and depression $[10,11]$. Emotional disorders and impaired information processing ability accelerate into decisional fatigue, which influences the ability of decisional judgement [12]. In addition, ICU familyclinician consultation usually with a simple and nonstandard oral-way, which will result in a knowledge gap. A survey study shows the discordance of predicting prognosis among clinicians and surrogates. More about $80 \%$ surrogates overestimate the prognosis of RRT, although sufficiently communication and mostly understanding [13]. Obviously, several uncertainties and risks exit in ICU patients this particular population [14-16], which makes it difficult for surrogates to comprehend the overall prognosis of RRT $[17,18]$. Further intervention is needed to make surrogates scientifically and efficiently understand the prognosis so as to narrow this knowledge gap. To sum up, we need to pay more attention to surrogates in decision-making engagement for ICU patients with AKI who requiring RRT.

For family surrogates and ICU clinicians to reach the decisional concordance, verified decision aids are needed to facilitate shared decision-making in ICU. Shared decision-making (SDM) refers to the interaction of patients or families with healthcare professionals to weigh the pros and cons of treatment options, and attaches importance to patients' preferences and values for reasonably predicting the best interests of patients [19]. International guidelines recommend that critical care specialists should discuss the treatment with patients and/or their families as soon as possible following SDM, when AKI patients have indications for RRT [20]. Decision aid (DA) is designed to inform patients and their families about treatment options, prognosis and possible decision outcomes, to help decide which choice is preferred after evaluation of the desirability and attributes of treatment options [21]. A Cochrane systematic review shows that DA could effectively improve the decision quality based on the evidence and a value, without increase the anxiety or depression of decision makers or worse patient and health system outcomes [22]. Stacey's research team has pointed that DA was associated with a greater knowledge of options, an increased rate of selecting with patients' values, reducing decisional conflict and higher patient participation [21]. However, there is a lack of decision supports like DA for family surrogates of ICU patients with AKI who requiring RRT.

In order to cope this complex decisional dilemma, the present study aims to: 1) develop a decision aid for family surrogate decision makers of ICU patients with AKI who requiring RRT guided by a systematic and rigorous process; 2 ) test the comprehensibility, acceptability and usability of the decision aid in a 
simulated decisional context ("near-live"); 3) verify the feasibility of the decision aid in ICU clinical practice ("live").

\section{Methods}

To develop the decision aid, a systematic development process model [23] will be conducted in combination with user-centered design (UCD) [24]. This study will be conducted in three phases. The whole development of decision aid prototype will be presented in phase 1 . The user-testing study for revision will be conducted in phase 2 and phase 3, with alpha-testing in a simulated way and beta-testing in the clinical context respectively. This study follows the IPDASi v4.0 [25] to meet the minimum quality criteria at least. The main elements of this development process illustrate in Fig. 1.

Figure 1 Development process of decision aid

A multidisciplinary steering committee to guide the whole development is significant. Therefore, a steering committee was created firstly to ensure a systematic protocol, which consists of two experts in SDM, three in critical care medicine and two patient representatives. Two SDM experts have many years of research experiences in shared decision making and patient decision aids. These critical care experts include an ICU director, a head nurse and a critical care specialist with expertise in RRT decision-making and clinician-family consultation. Our patient representatives are family surrogate volunteers who experienced RRT decision-making in ICU. Steering committee meetings will be hold regularly to supervise the design, content and iterative revision of the decision aid.

Phase 1: Development of decision aid prototype

Scope: literature review

A comprehensive literature review occurs early to scope the purpose of decision aid development. Firstly, extensive literature reviews will be performed to understand the current state of RRT decision-making and identify the evidence-based necessity to develop a DA in ICU. In addition, we will conduct a competitive analysis by summarizing relevant DA resources, which is an important way for digital projects development, to determine the development value rather than duplicating the available contributions. Furthermore, this is a significant step to ensure whether the development project is compatible with our research protocol.

Design: needs assessment

\section{Healthcare professionals' interviews}

Concurrent with the literature review, decisional needs assessment is critical in confirming potential DA use cases as identified in literature. In terms of the complexity (as one of the life-sustaining treatment) of RRT decision-making in ICU, the decision trigger always opts patients' attending clinician. Therefore, semi-structured interviews were conducted with ICU clinicians to identify main points that a DA might 
address and opportunities to integrate DA into clinical workflow. We will recruit ICU clinicians who recently ( $<6$ month ago) went through clinician-family consultations about RRT decision-making for patients with AKI. Each interview will typically last 30 and 60 minutes, and aims to elicit professional opinions on key aspects in content, design and distribution of DA. Firstly, clinicians will be asked to indicate what information family surrogates needed to know before joining in such decision-making. Secondly, they will be asked to outline the surrogate decision-making pathway to dig out when and how to refer to a DA is appropriate. Accordingly, individual interviews with ICU clinicians are to explore professional suggestions and reach consensus on their clinical needs for this DA.

All interviews will be recorded and transcribed verbatim. The interviewee's extra perspectives will be written down by the interviewer, then analyzed alongside with transcripts. Colaizzi seven-step qualitative analysis approach will be used for coding emerging categories and themes in transcripts [26]. Firstly, all transcripts will be independently reviewed and coded by two researchers with expertise in qualitative research methods. Then, final coding will be determined by further comparison and discussion, and disagreement of opinions will be submitted to another qualitative research expert for arbitration. Computer software NVivo version 12 will be used for analysis.

\section{Target users' interviews}

UCD requires a deep understanding of target user's role and responsibility related to the DA in development. To comply with this requirement, we will conduct semi-structured interviews with family surrogates, the potential target user of the DA, who are informed with and engaged in the currently RRT decision-making for their loved one. For generating a comprehensive concept regarding to surrogate decision-making in RRT, site observations especially when clinician-family encounters happen in ICU routine are needed. Therefore, we will timely focus the ICU clinician-family consultation before face-toface interviews with surrogates to uncover the opportunity that a DA can fulfill their decisional needs. Opportunity statement exercise[24] will be used, which is a type of design thinking activity used to gather, explore and refine the information needed for decision aid development, bringing together with family surrogates to collect delineating facets of current practice that are not meeting surrogate decisional needs to identify in what way a DA can make measurable impact. Finally, family surrogates will be asked to provide feedback on prepared statements (summary outcome of literature review and healthcare professionals' interviews), which describe a future decision aid and its general contents, formats and functionalities. Similar to the analysis of interviews among ICU clinicians, all of the audio recordings will be transcribed verbatim and analyzed along with written notes using qualitative analysis approach.

Then, the decisional needs from both ICU clinicians and family surrogates will be translated into a general framework for next-stage detailed evidence synthesis.

Draft: working prototype

Evidence synthesis 
The best evidence and other decisional information will be synthesized based on the needs assessments between ICU clinicians and surrogates. Firstly, we will review current guidelines on the management of AKI and guidance of RRT for general aspects of treatment recommendations. Secondly, available treatment-related outcome evidences from systematic reviews and meta-analysis will be systematically searched for generating suitable information for users concerning whether or not to choose RRT. The whole process of evidence synthesis will be as following four steps: 1) identify the key words; 2) systematically searching; 3 ) screen and extract relevant evidences based on clinical needs, usually about treatment recommendations and prognostic information; 4) sum up evidences into a decision aid script.

\section{Drafting the prototype}

According to literature review, key stakeholders' needs assessment and best available evidences, a paperformat prototype will be drafted under the guidance with several criteria for development of decision aids $[27,28]$ and assessed following the IPDASi v4.0. In general, the content of the DA will be presented in five aspects: 1 ) brief instruction of disease and treatment; 2 ) pros and cons of treatment options; 3 ) possible decisional outcomes; 4) patient story; 5) value clarification exercise. Once the paper-format prototype has been completed, the steering committee will audit and decide the final content included in the decision aid. Then, the paper-format prototype will be redrafted and redesigned into a working prototype which can be accessed by scanning Quick Response Code (QR code) using smart phone.

\section{Phase 2: Alpha-testing}

The aim of this phase is to examine the comprehensibility, acceptability and usability of the DA in a simulated decision-making scenario,[23] for a better understanding of the interaction between decision aids and target users in conducting tasks. This testing will be done in surrogates, requiring interaction with the DA and carrying out think-aloud method. The evaluation objectives include assessment of the functionality and usability of the DA's overall interface and identifying problems in user interaction.

\section{Participants}

A purposive sample of surrogates not involved in development process will be invited into participate. We will recruit family surrogate decision-makers of AKI patients from Southwest hospital in Chongqing, China. The surrogate samples will include those who opted RRT for their loved one, those who considered but declined RRT, and those currently deliberating treatment options in ICU. We expect a sample size of 812 participants, to detect up to $80 \%$ of the surface level usability problems[29]. However, sample sizes will be determined by data saturation, which is guided by transcripts of users' interaction with the aid. We will conduct and complete alpha-testing within four weeks.

Data collection

A researcher with expertise in health informatics and usability testing will guide each session in a quiet waiting room. Data will be collected during the whole process of participants' usage of the DA, including questionnaires, recording of the interaction and semi-structured interviews. Firstly, participants will be 
required to complete the before-questionnaire related to their demographics, self-perceived on-line information acquiring skills on smart phone and experiences about RRT decision-making, and a knowledge survey [30] about general RRT information. Secondly, participants will be subsequently presented a scenario describing a simulated decision making about RRT for AKI patients in ICU, which is closely matching their history experiences. Then, they will be given an introduction card containing the retrievable address (a QR code scanned using WeChat on smart phone) of the $D A$, and also a brief task description that participants need to complete the usage of the DA with think-aloud method, which means oral expressing their behaviors and thoughts while using. With the task completed, participants will be required to finish the after-questionnaire, including a knowledge survey like before and an acceptability survey [31]. Finally, participants will be interviewed regarding satisfaction, pros and cons of the DA and the quality of this decision support. We will open screen-recording on the phone to typically record the whole user interaction (all screen activities and voice). Field notes will be written for expanding data sources as further analysis.

Data analysis

In keeping with effective sample size, data analysis will occur in conjunction with data collection. Data analysis will be conducted with a coding scheme developed by usability engineering team from examination of human-computer interactions and relevant cognitive literatures[29]. This scheme consists of eleven categories of usability problems, such as navigation, graphics, layout and so on. It allows application of these available categories in analyzing user interaction with the DA along with think-aloud reports. However, codes that indicate other user problems generated from our testing results will be added appropriately.

The screen recordings will be watched and annotated independently by two researchers. Disagreement will be discussed to reach a consensus. Semi-structured interviews will be analyzed as described in key stakeholders' interviews in Phase 1. Knowledge surveys will be analyzed by paired t-test. Acceptability surveys will be analyzed by one-sample t-test. SPSS Statistics version 26 will be used for analysis.

Based on these findings, refined details for improvement will be generated. These efforts will be reviewed by steering committee to decide following revision.

\section{Phase 3: Beta-testing}

Beta-testing will be conducted to test the feasibility of the DA in ICU clinical context, for considering contextual factors which may influence the effect of the DA in real life. Without developers' control, this stage is a more stable acceptance testing. A mixed-methods approach will be used to conduct a before/after study in RRT decision making procedure. The objectives of beta-testing are to assess the impact of the DA on family surrogate engagement in RRT decision making and the quality of decision making.

Participants 
Both family surrogate decision makers and ICU clinicians will be recruited from ICU clinical workflow in the same hospital as described in alpha-testing. We will recruit participants based on patients. If AKI patients indicated with RRT requirement, we will first recruit clinicians to ensure the following familyclinician consultation for decision making. Then, we will invite each family surrogate to participate in. We will take the criteria, that who is responsible for written informed consent for patients' medical decision making, as the surrogate standard. Clinicians needed to be with at least three years of ICU clinical experiences, and are responsible for guiding the decision-making process. Clinicians who are reluctant for shared decision making with patients' family will be excluded. Family surrogates who are not able to use general function of WeChat on a smart phone will be excluded. Either clinician or surrogate is not eligible, patient will be excluded.

A consecutive sample of 15-20 family-clinician dyads will be invited to participate, which is in line with previous study in order to conduct inferential statistics in usability testing[29]. We are proposing to recruit more participants as soon as possible, to generate sufficient feedback for further refinement.

\section{Data collection}

Once the family-clinician dyad is available, DA will be introduced to surrogates before the decisionmaking consultation occurs. After completion of the usage, family surrogates will be invited into a formal consultation with clinicians for their loved one's treatment decision making. All eligible surrogates need to complete questionnaire survey before and after using the DA, which includes demographics, surrogate decision making self-efficacy scale[32], preparation of decision making scale[33] and decisional conflict scale[34]. After the consultation, family surrogates and clinicians will be interviewed independently by two interviewers respectively, for providing verbal feedback on the process of decision making. Family surrogates will be asked for description of whether or not the DA facilitates engagement in decision making and meets their decisional needs. Clinicians will be required for sharing their experiences in this kind of decision making, and evaluating the decision quality. Interview outlines for surrogates and clinicians will be respectively prepared in advance and approved in steering committee meeting. All interviews will be recorded and last 30 45 minutes. All sessions will be undertaken in a quiet waiting room.

Data analysis

Descriptive analysis will be conducted for reporting baseline data. Paired sample t-test will be performed to assess self-efficacy, preparation and decisional conflict. Cls will be set at $95 \%$, and significant level is $5 \%$. All interviews will be transcribed and coded using NVivo version 12.0, and analyzed in line with the methodology in phase 1. Based on these findings, we will refine the aid if necessary. Finally, the steering committee will confirm the completion of development as a final version waiting for next-stage formal clinical trial.

Patient and public involvement 
Two family surrogate decision makers were recruited as patient representatives in the steering committee. These two patient representatives will narrate their decision-making stories, which is anticipated to contribute participants heavily to clarify their loved one's preferences. Patient representatives will engage in the whole development to focus on the content and design of the aid. We expected it would be user-centered for the presentation of our decision aid in the user's perception. All of these representatives will not contribute to the recruitment.

Ethics and dissemination

Research ethics approval from Ethics Committee of the First Affiliated Hospital of Army Medical University, PLA (Ref: KY2020104) has been granted. Study statement information will be given to each participant. Written informed consent will be obtained from all participants. Participants will have the right to withdraw at any time of this study. All the important original innovative data will be considered for publication in peer-reviewed journals.

\section{Discussion}

This study introduces the rigorous development and evaluation of a decision aid for family surrogate decision makers of critically ill patients with AKI who are requiring RRT. We expect to address the objective of improving SDM and the quality of RRT decision-making in ICU. Over the past two decades, SDM has been introduced in China, but now public attention bounded with paternal model in medical decision-making has hardly changed. Actually, there are more theoretical studies than empirical researches in clinical practice. Decision-making still dominated by ICU clinicians [35, 36], and patients' especially family surrogates' attitudes and decision-making engagements need to be explored in China. The State Council issued an outline for the "Healthy China 2030" initiative [37], which indicates that the patients' sense of access to medical treatment should be enhanced during treatment procedure optimization. It also proposes to strengthen humanistic care in medical service to build a harmonious doctor-patient relationship. Medical decision making led by paternal model can no longer adapt to modern medical services in China today, and shared decision making is needed to achieve best decision making for both patients and healthcare providers.

In any case, there is a lack of implementation of SDM in RRT decision making in worldwide ICU. A decision-making process called "shared" if the SDM behavior is shown by both clinicians and patients or surrogates, sense of participation gained by patients or surrogates, and sharing views and ideas together to get the decisional concordance [38]. However, available data shows that the discordance of RRT decision-making between clinicians and surrogates exists [9]. Furthermore, surrogates are usually unable to reasonably estimate the prognosis of their loved one, although with a sufficiently communication and understanding [13].

User-centered design in health IT tools can especially for users to gripe convoluted process and inaccessible data [24]. The goal of this decision aid, which involves target users' participation in development stage to concord with user-centered, is to increase surrogate's SDM ability by helping them 
better involvement in clinician-family consultation. Indeed, we think that this decision aid will improve understanding of $\mathrm{AKI}$ and relevant therapy options, risks and benefits of treatment, possible decisional outcomes, and the importance of patient's preferences and values. We also expect that this decision aid will improve surrogate decision making engagement by reducing decisional stress and other negative emotions.

\section{Abbreviations}

AKI: Acute kidney injury; RRT:Renal replacement therapy; SDM:Shared decision making; DA:Decision aid; UCD:User-centered design; IPDASi v4.0:International patient decision aid standards instrument version 4.

\section{Declarations}

\section{Ethical Approval and Consent to participate}

Ethics approval for this study was provided by the Ethics Committee of the First Affiliated Hospital of Army Medical University, PLA (Ref: KY2020104) on June 24, 2020. All participants will sign formal informed consent.

\section{Consent for publication}

Not applicable.

\section{Availability of supporting data}

Data sharing is not applicable to this article as no datasets were generated or analyzed during the current study.

\section{Competing interests}

The authors declare that they have no competing interests.

\section{Funding}

The study is funded by Chongqing Social Science Planning Youth Project (2018QNSH42) and Chongqing Postgraduate Education and Teaching Reform Project (yjg172013), which had no role in the design, collection, analysis nor interpretation of data, nor in the writing nor publication of the manuscript.

\section{Authors' contributions}

ZM conceived of the study and drafted the manuscript. All authors participated in the design of the study, revised critically for intellectual content, and have read and approved the final manuscript.

\section{Acknowledgements}


Special thanks to the patient representatives. We thank the participants who volunteered to take part in this research.

\section{References}

1. Yang L, Xing G, Wang L, Wu Y, Li S, Xu G, et al. Acute kidney injury in China: a cross-sectional survey. The Lancet. 2015;386:1465-71.

2. Jiang L, Zhu Y, Luo X, Wen Y, Du B, Wang M, et al. Epidemiology of acute kidney injury in intensive care units in Beijing: the multi-center BAKIT study. BMC Nephrol. 2019;20:468.

3. Rachoin JS, Weisberg LS. Renal Replacement Therapy in the ICU. Crit Care Med. 2019;47:715-21.

4. Ronco C. Continuous renal replacement therapy: forty-year anniversary. Int J Artif Organs. 2017;40:257-64.

5. Uchino S, Kellum JA, Bellomo R, Doig GS, Morimatsu H, Morgera S, et al. Acute renal failure in critically ill patients: a multinational, multicenter study. JAMA. 2005;294:813-8.

6. Zhao Y, Chen Y. Effect of renal replacement therapy modalities on renal recovery and mortality for acute kidney injury: A PRISMA-compliant systematic review and meta-analysis. Semin Dial. 2020;33:127-32.

7. Karkar A, Ronco C. Prescription of CRRT: a pathway to optimize therapy. Annals of Intensive Care. 2020;10:32.

8. Butler CR, O'Hare AM. Complex Decision Making about Dialysis in Critically III Older Adults with AKI. Clin J Am Soc Nephrol. 2019;14:485-7.

9. Bagshaw SM, Adhikari NKJ, Burns KEA, Friedrich JO, et al Selection and Receipt of Kidney Replacement in Critically III Older Patients with AKI. Clin J Am Soc Nephrol. 2019, 14:496-505.

10. Wendlandt B, Ceppe A, Choudhury S, Cox CE, Hanson LC, Danis M, et al. Modifiable elements of ICU supportive care and communication are associated with surrogates' PTSD symptoms. Intensive Care Med. 2019;45:619-26.

11. Pochard Fdr, Azoulay E, Chevret S, Lemaire Fo, Hubert P, Canoui P, et al. Symptoms of anxiety and depression in family members of intensive care unit patients- Ethical hypothesis regarding decisionmaking capacity. Crit Care Med. 2001;29:1893-7.

12. Hickman RL, Pignatiello GA, Tahir S. Evaluation of the Decisional Fatigue Scale Among Surrogate Decision Makers of the Critically III. West J Nurs Res. 2018;40:191-208.

13. Allegretti AS, Hundemer G, Chorghade R, Cosgrove K, Bajwa E, Bhan I. Perspectives of continuous renal replacement therapy in the intensive care unit: a paired survey study of patient, physician, and nurse views. BMC Nephrol. 2015;16:105.

14. Zarbock A, Kellum JA, Schmidt C, Van Aken H, Wempe C, Pavenstadt H, et al. Effect of Early vs Delayed Initiation of Renal Replacement Therapy on Mortality in Critically III Patients With Acute Kidney Injury: The ELAIN Randomized Clinical Trial. JAMA. 2016;315:2190-9. 
15. Barbar SD, Clere-Jehl R, Bourredjem A, Hernu R, Montini F, Bruyère R, et al. Timing of RenalReplacement Therapy in Patients with Acute Kidney Injury and Sepsis. N Engl J Med. 2018;379:1431-42.

16. Gaudry S, Hajage D, Schortgen F, Martin-Lefevre L, Pons B, Boulet E, et al. Initiation Strategies for Renal-Replacement Therapy in the Intensive Care Unit. N Engl J Med. 2016;375:122-33.

17. Wu L, Zhang P, Yang Y, Jiang H, He Y, Xu C, et al. Long-term renal and overall survival of critically ill patients with acute renal injury who received continuous renal replacement therapy. Ren Fail. 2017;39:736-44.

18. De Corte W, Dhondt A, Vanholder R, De Waele J, Decruyenaere J, Sergoyne V, et al. Long-term outcome in ICU patients with acute kidney injury treated with renal replacement therapy: a prospective cohort study. Crit Care. 2016;20:256.

19. Charles C, Gafni A, Whelan T. Shared Decision-Making in the Medical Encounter: What Does It Mean? (Or It Takes at Least Two to Tango). Soc Sci Med. 1997;44:681-92.

20. Guideline Updates Team (UK). Acute kidney injury: prevention, detection and management. London: National Institute for Health and Care Excellence (UK); 2019.

21. Stacey D, Légaré F, Lewis KB. Patient Decision Aids to Engage Adults in Treatment or Screening Decisions. JAMA. 2017;318:657-8.

22. Stacey D, Légaré F, Lewis K, Barry MJ, Bennett CL, Eden KB, et al. Decision aids for people facing health treatment or screening decisions (Review). Cochrane Database Syst Rev. 2017:CD001431.

23. Coulter A, Stilwell D, Kryworuchko J, Mullen PD, Ng CJ. Weijden Tvd. A systematic development process for patient decision aids. BMC Med Inform Decis Mak. 2013;13:2.

24. Chokshi SK, Mann DM. Innovating From Within: A Process Model for User-Centered Digital Development in Academic Medical Centers. JMIR Human Factors. 2018;5:e11048.

25. Joseph-Williams N, Newcombe R, Politi M, Durand MA, Sivell S, Stacey D, et al. Toward Minimum Standards for Certifying Patient Decision Aids: A Modified Delphi Consensus Process. Med Decis Making. 2014;34:699-710.

26. Colaizzi P. Psychological research as the phenomenologist views it. New York: Oxford University Press; 1978.

27. Trevena LJ, Zikmund-Fisher BJ, Edwards A, Gaissmaier W, Galesic M, Han PK, et al. Presenting quantitative information about decision outcomes: a risk communication primer for patient decision aid developers. BMC Med Inform Decis Mak. 2013;13:7.

28. Abhyankar P, Volk RJ, Blumenthal-Barby J, Bravo P, Buchholz A, Ozanne E, et al. Balancing the presentation of information and options in patient decision aids: an updated review. BMC Med Inform Decis Mak. 2013;13:6.

29. Kushniruk AW, Patel VL. Cognitive and usability engineering methods for the evaluation of clinical information systems. J Biomed Inform. 2004;37:56-76. 
30. O'Connor AM. User Manual - Knowledge[document on the Internet] Ottawa: Ottawa Hospital Research Institute. http://decisionaid.ohri.ca/docs/develop/User_Manuals/UM_Knowledge.pdf. Accessed 21 July 2020.

31. O'Connor AM, Cranney A. User Manual - Acceptability[document on the Internet] Ottawa: Ottawa Hospital Research Institute.

http://decisionaid.ohri.ca/docs/develop/User_Manuals/UM_Acceptability.pdf. Accessed 21 July 2020.

32. Lopez RP, Guarino AJ. Psychometric Evaluation of the Surrogate Decision Making Self-Efficacy Scale. Res Gerontol Nurs. 2013;6:71-6.

33. Bennett C, Graham ID, Kristjansson E, Kearing SA, Clay KF, O'Connor AM. Validation of a Preparation for Decision Making scale. Patient Educ Couns. 2010;78:130-3.

34. O'Connor AM. Validation of a Decisional Conflict Scale. Med Decis Making. 1995;15:25-30.

35. Huang C, Plummer V, Lam L, Cross W. Shared decision-making in serious mental illness: A comparative study. Patient Educ Couns. 2020.

36. Zhai H, Lavender C, Li C, Wu H, Gong N, Cheng Y. Who decides? Shared decision-making among colorectal cancer surgery patients in China. Support Care Cancer. 2020.

37. The State Council of the People's Republic of China. Healthy China. 2030 initiative. 2016. http://www.gov.cn/xinwen/2016-10/25/content_5124174.htm. Accessed 22 July 2020.

38. Geiger F, Liethmann K, Hoffmann F, Paschedag J, Kasper J. Investigating a training supporting Shared Decision Making (IT'S SDM 2011): study protocol for a randomized controlled trial. Trials. 2011;12:232.

\section{Figures}

Development process of decision aid

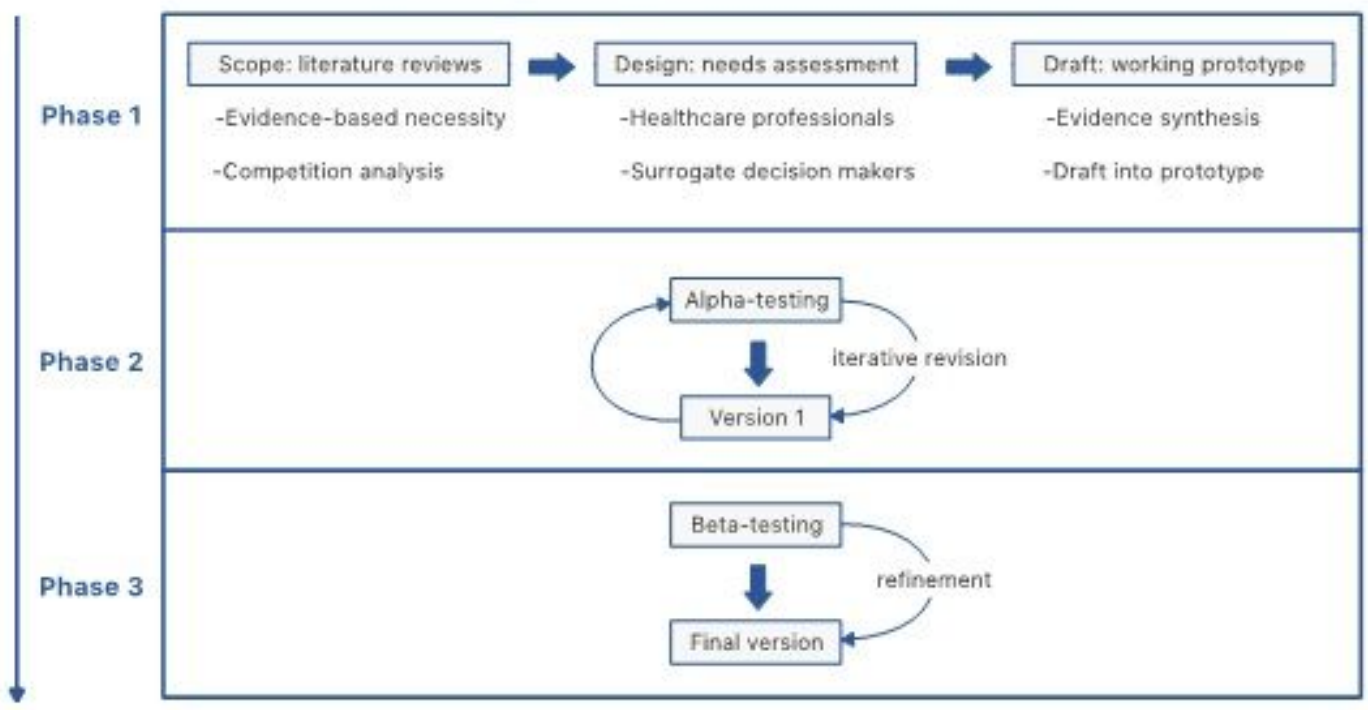

Figure 1 
Development process of decision aid

\section{Supplementary Files}

This is a list of supplementary files associated with this preprint. Click to download.

- renameddebb3.pdf

- renameddebb3.pdf

- fundingletter.pdf

- fundingletter.pdf 